OPEN ACCESS

Edited by:

Paolo Bianchini,

Italian Institute of Technology, Italy

Reviewed by:

Yiqing Lu,

Macquarie University, Australia

Stefania Abbruzzetti,

University of Parma, Italy

*Correspondence:

Sanghee Lee

slee19@kist.re.k

Yong II Park

ypark@jnu.ac.kr

${ }^{\dagger}$ These authors have contributed equally to this work

Specialty section:

This article was submitted to

Nanobiotechnology,

a section of the journal

Frontiers in Bioengineering and

Biotechnology

Received: 10 January 2020

Accepted: 16 March 2020

Published: 16 April 2020

Citation:

Lee SY, Lee R, Kim E, Lee S and

Park YI (2020) Near-Infrared

Light-Triggered Photodynamic

Therapy and Apoptosis Using Upconversion Nanoparticles With

Dual Photosensitizers.

Front. Bioeng. Biotechnol. 8:275.

doi: 10.3389/fbioe.2020.00275

\section{Near-Infrared Light-Triggered Photodynamic Therapy and Apoptosis Using Upconversion Nanoparticles With Dual Photosensitizers}

\author{
Song Yeul Lee ${ }^{1 \dagger}$, Ruda Lee't, Eunha Kim³, Sanghee Lee ${ }^{4 *}$ and Yong II Park ${ }^{1 *}$ \\ 'School of Chemical Engineering, Chonnam National University, Gwangju, South Korea, ${ }^{2}$ International Research \\ Organization for Advanced Science and Technology, Kumamoto University, Kumamoto, Japan, ${ }^{3}$ Department of Molecular \\ Science and Technology, Ajou University, Suwon, South Korea, ${ }^{4}$ Center for Neuro-Medicine, Brain Science Institute, Korea \\ Institute of Science and Technology, Seoul, South Korea
}

Elucidation of upconversion nanoparticles (UCNPS) that can be excited by nearinfrared (NIR) light is an interesting topic in the field of photodynamic therapy (PDT). However, the PDT efficiency of conventional UCNPs is limited due to the low quantum yield and overheating effect of the $980 \mathrm{~nm}$ light source. In this study, a light source with a wavelength of $808 \mathrm{~nm}$ was used as an excitation source for $\mathrm{Nd}$ doped UCNPs to solve the overheating effect. UCNPs with a core@shell structure $\left(\mathrm{NaYF}_{4}: \mathrm{Yb}, \mathrm{Er}, \mathrm{Nd} @ \mathrm{NaYF}_{4}: \mathrm{Yb}, \mathrm{Nd}\right.$ ) were synthesized to increase the upconversion emission efficiency. Dual-color emitting Er-doped UCNPs and dual photosensitizers (Chlorin e6 and Rose Bengal) were used for enhanced PDT. Each photosensitizer could absorb red and green emissions of the UCNPs to generate reactive oxygen species (ROS), respectively. The ROS generation in a dual photosensitizer system is significantly higher than that in a single photosensitizer system. Additionally, PDT induces immunogenic apoptosis. In this study, by utilizing a highly efficient PDT agent, PDT-induced apoptosis was studied by biomarker analysis.

Keywords: near-infrared, upconversion, nanoparticle, photodynamic therapy, apoptosis

\section{INTRODUCTION}

Various anticancer therapies are currently being tested in clinics. However, new cancer remedies are still needed for selective and efficient treatment with minimal side effects. Photodynamic therapy (PDT) is one such method where photosensitizer molecules are excited by light to produce cytotoxic reactive oxygen species (ROS) to kill tumor cells (Dolmans et al., 2003). PDT is a minimally invasive therapeutic modality when compared to traditional surgical treatments. Light-induced local PDT 
treatment reduces the side effects of normal cell death by administering non-specific anticancer drugs. PDT is also known to induce an immune response by damaged tumor cells, which releases tumor-associated antigens and elicit antitumor immune responses (Maeding et al., 2016; Yang et al., 2016). PDT is usually used to treat local lesions by irradiation with light and the induction of immune responses opens the possibility of treating metastatic tumors or distant sites that cannot be reached by light (Zheng et al., 2016; Xu et al., 2017a; Hwang et al., 2018; Yan et al., 2019).

The PDT requires highly efficient photosensitizers to produce cytotoxic ROS. However, conventional photosensitizers used in PDT are usually excited by visible light, which limits the penetrating depth of the light source in tissues. Therefore, to increase the light penetration depth, it is necessary to develop efficient PDT agents that are excited by long wavelengths, such as near-infrared (NIR) light (Kobayashi and Choyke, 2019). Recently, lanthanide-doped upconversion nanoparticles (UCNPs) were developed to achieve NIR-triggered PDT (Guo et al., 2010; Tsai et al., 2018). As an anti-Stokes shifting material, UCNPs are the most efficient materials for converting NIR light to UV and visible light (Chen et al., 2014; Dong et al., 2015). Therefore, UCNPs can be used as a transducer to activate a conventional photosensitizer that is sensitive to visible light by using a NIR light source. Additionally, the use of NIR photons minimizes phototoxicity and background autofluorescence (Park et al., 2009; Wu et al., 2009), which is beneficial for bioimaging, diagnosis, and therapy. UCNP-based theranostic agents have been reported for simultaneous diagnosis and treatment of diseases (Park et al., 2012; Feng et al., 2019; Huang et al., 2020).

In this study, NIR-responsive PDT agents with dual photosensitizers were prepared. The $808 \mathrm{~nm}$ wavelength excitation was selected instead of the $980 \mathrm{~nm}$ wavelength to minimize overheating by the light source (Wang et al., 2013). For efficient photosensitizer activation using the $808 \mathrm{~nm}$ NIR wavelength excitation, $\mathrm{NaYF}_{4}: \mathrm{Yb}, \mathrm{Er}, \mathrm{Nd} @ \mathrm{NaYF}_{4}: \mathrm{Yb}, \mathrm{Nd}$ was selected as Nd-doped UCNPs with a core@shell structure (Huang and Lin, 2015). The dual photosensitizers, Chlorin e6 and Rose Bengal, were used to activate the red and green emission of UCNPs. The dual photosensitizer system showed a synergistic ROS generation, as compared to the single photosensitizer system (Idris et al., 2012; Xu et al., 2017b; Yang et al., 2019). The ROS production achieved by NIR and PDT-induced apoptosis was investigated with regard to cellular studies.

\section{EXPERIMENTAL SECTION}

\section{Synthesis of Photosensitizer-Loaded Upconversion Nanoparticles Synthesis of $\mathrm{NaYF}_{4}: \mathrm{Yb}, \mathrm{Er}, \mathrm{Nd}$ Core UCNPs}

The UCNPs were synthesized using a previously reported method with slight modifications (Huang and Lin, 2015). For $\mathrm{NaYF}_{4} \mathrm{Yb}, \mathrm{Er}, \mathrm{Nd}$ core UCNPs, $0.795 \mathrm{mmol}$ of yttrium acetate hydrate (99.9\%), $0.19 \mathrm{mmol}$ of ytterbium acetate hydrate (99.9\%), $0.005 \mathrm{mmol}$ of erbium acetate hydrate (99.9\%), and
$0.01 \mathrm{mmol}$ of neodymium acetate hydrate (99.9\%) were added into a three-necked flask, and mixed with $11.84 \mathrm{~g}$ of oleic acid (OA, technical grade, 90\%) and $5.32 \mathrm{~g}$ of 1-octadecene (ODE, technical grade, 90\%). The residual water in the reaction mixture was removed at $150^{\circ} \mathrm{C}$ after $40 \mathrm{~min}$. After cooling the temperature to $100^{\circ} \mathrm{C}$, a methanol solution containing $0.1 \mathrm{~g}$ of $\mathrm{NaOH}$ (97\%) and $0.148 \mathrm{~g}$ of $\mathrm{NH}_{4} \mathrm{~F}$ (99.9\%) was added to the flask and stirred for $20 \mathrm{~min}$. The methanol was removed after $10 \mathrm{~min}$ at $110^{\circ} \mathrm{C}$ under vacuum. Then, the flask was heated to $310^{\circ} \mathrm{C}$ at a rate of $10^{\circ} \mathrm{C} / \mathrm{min}$ in an $\mathrm{Ar}$ atmosphere and the temperature was maintained for $60 \mathrm{~min}$. The core UCNPs were washed using ethanol and dispersed in cyclohexane.

\section{Synthesis of $\mathrm{NaYF}_{4}: \mathrm{Yb}, \mathrm{Er}, \mathrm{Nd} @ \mathrm{NaYF}_{4}: \mathrm{Yb}, \mathrm{Nd}$ corexs@shell UCNPs}

The core@shell UCNPs were synthesized by the epitaxial growth of the shell on the core nanoparticle surface. For the $\mathrm{NaYF}_{4}: \mathrm{Yb}, \mathrm{Nd}$ shell, $0.7 \mathrm{mmol}$ of yttrium acetate hydrate, $0.1 \mathrm{mmol}$ of ytterbium acetate hydrate, and $0.2 \mathrm{mmol}$ of neodymium acetate hydrate were mixed in a three-necked flask with $11.84 \mathrm{~g}$ of $\mathrm{OA}$ and $5.32 \mathrm{~g}$ of ODE. After removing the residual water at $150^{\circ} \mathrm{C}$, the flask was cooled to $70^{\circ} \mathrm{C}$. A solution of core UCNPs in cyclohexane was added to the flask. The remaining synthetic procedure of the core@shell UCNPs was similar to that of the core UCNPs. The size and shape of the UCNPs were measured using transmission electron microscopy (TEM, JEOL-2100F, Japan), and the crystalline structures were recorded using an X-ray diffractometer (XRD, Rigaku, Rint 1000, Japan). The emission spectra of UCNPs were measured using a spectrophotometer (FluoroMax-4, Horiba) equipped with an $808 \mathrm{~nm}$ continuous wave (CW) laser (Opto Engine LLC). An inductively coupled plasma atomic emission spectrophotometer (ICP-AES) was used to determine the concentration of UCNPs.

\section{Surface Modification of UCNPs}

To impart water dispersibility, as-synthesized hydrophobic UCNPs were encapsulated with PEG-phospholipids (1,2distearoyl-sn-glycero-3-phosphoethanolamine-N-[methoxy (polyethylene glycol)-2000], DSPE-PEG, Avanti Polar Lipids, Inc.) (Park et al., 2012). The UCNPs were mixed with excess DSPE-PEG in $10 \mathrm{~mL}$ of chloroform. After the complete removal of chloroform, the DSPE-PEG encapsulated UCNPs were redispersed in water. The excess DSPE-PEG was removed by repeated centrifugation (13,200 rpm for $20 \mathrm{~min})$.

\section{Loading Photosensitizers on the DSPE-PEG Encapsulated UCNPs}

The DSPE-PEG encapsulated UCNPs were mixed with a photosensitizer solution containing $1 \mu \mathrm{mol}$ of chlorin e6 (Ce6, Frontier Scientific, Inc.) or rose bengal (RB, SigmaAldrich). Depending on the photosensitizer loaded, four types of samples (e.g., UCNPs, RB-UCNPs, Ce6-UCNPs, and RB/Ce6UCNPs) were prepared. The mixture was wrapped with an $\mathrm{Al}$ foil and incubated overnight at room temperature. The unloaded photosensitizers were removed by centrifugation, and 

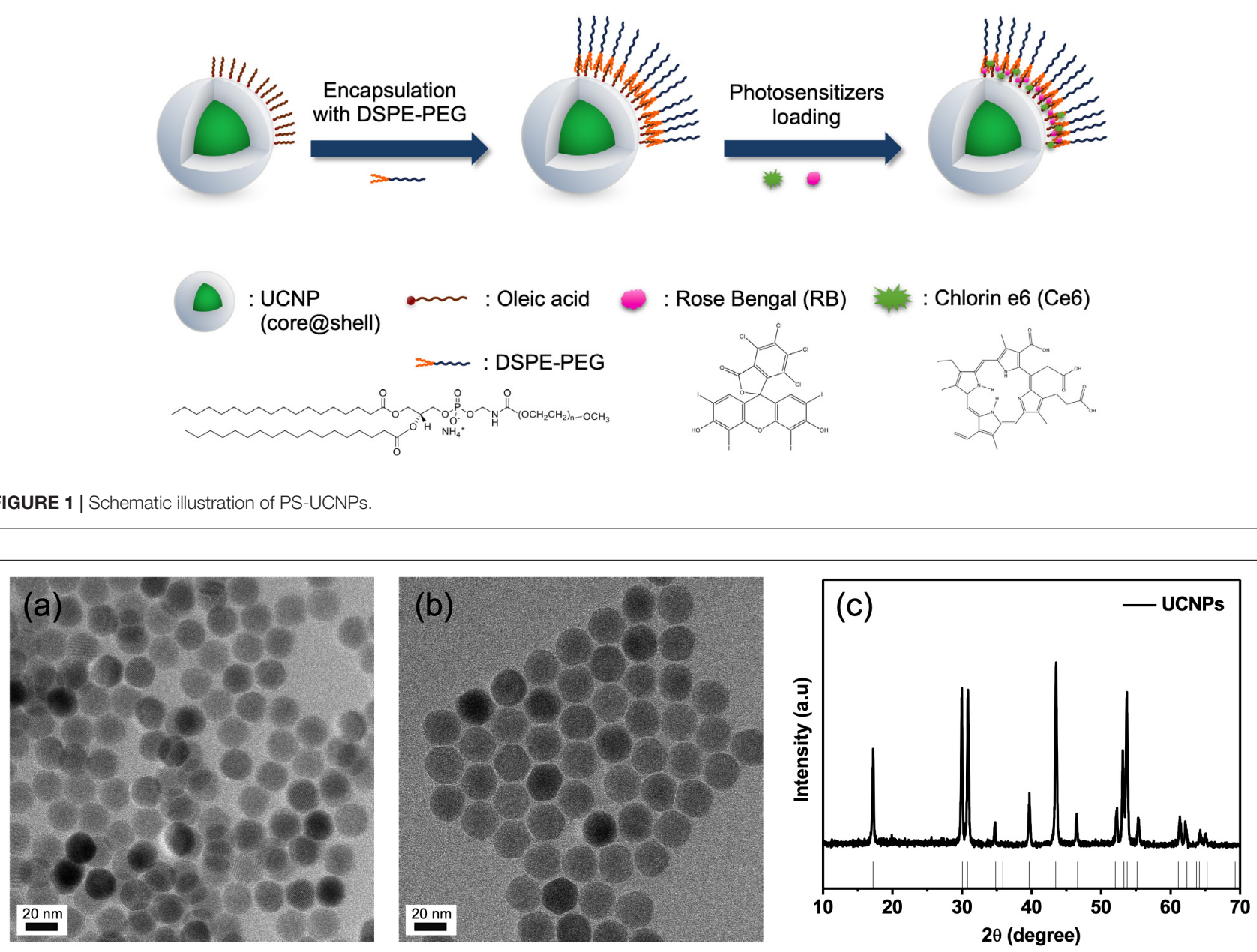

FIGURE 2 | Characterization of UCNPs. (a) TEM image of core UCNPs, (b) TEM image of core@shell UCNPs, and (c) XRD patterns of core@shell UCNPs.
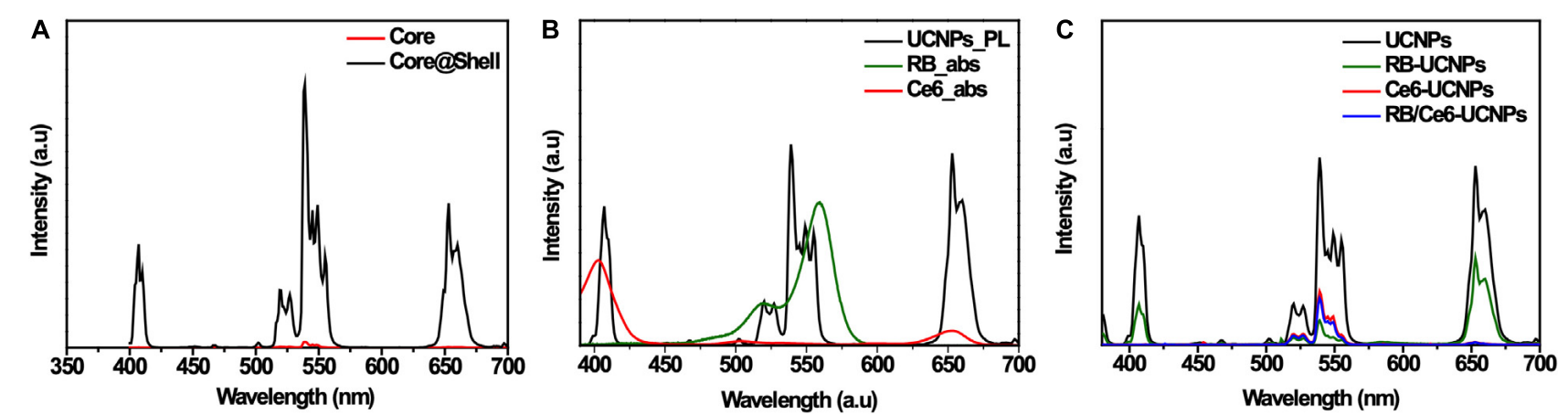

FIGURE 3 | Optical properties of UCNPs and PS-UCNPs. (A) Photoluminescence (PL) spectra of core UCNPs and core@shell UCNPs. (B) Absorbance spectra of RB and Ce6, and PL spectrum of UCNPs. (C) PL spectra of four kinds of UCNP samples (Bare, RB-loaded, Ce6-loaded, and RB and Ce6-loaded samples).

the photosensitizers-loaded UCNPs (PS-UCNPs) were further purified using the desalting column (PD 10, GE Healthcare). The number of PS per UCNPs were quantified by measuring the absorbance of the PS in UCNPs $\left(\sim 8 \times 10^{3} \mathrm{Ce} 6\right.$ and $\sim 1 \times 10^{3} \mathrm{RB}$ per RB/Ce6-UCNPs).

\section{Measurement of ROS Generation From PS-UCNPs}

A $10 \mu \mathrm{M}$ stock solution of 9,10-anthracenediylbis(methylene)dimalonic acid (ABDA, Sigma-Aldrich) was prepared in water. Then, $1 \mathrm{~mL}$ of the ABDA stock solution was 
added to $1 \mathrm{mg}$ of UCNPs loaded with different photosensitizers, respectively. The mixtures were irradiated using the $808 \mathrm{~nm} \mathrm{CW}$ laser (500 $\mathrm{mW}$, Opto Engine LLC). The decrease in fluorescence intensity of ABDA ( $\lambda_{\text {ex }} 380 \mathrm{~nm}$ and $\lambda_{\text {em }} 407 \mathrm{~nm}$ ) was measured using a spectrofluorometer (FluoroMax-4, Horiba).

\section{Cytotoxicity Assessment and Cellular PDT of PS-UCNPs}

The B16BL6 melanoma cells (ATCC) were cultured in DMEM containing $10 \%$ FBS and $1 \%$ penicillin/streptomycin in $5 \% \mathrm{CO}_{2}$ at $37^{\circ} \mathrm{C}$. The cells were seeded in a 96-well microplate at a density of $5 \times 10^{3}$ cells per well and incubated for $24 \mathrm{~h}$ (Xu et al., 2017a). The B16BL6 melanoma cells were treated with seven different concentrations (from 0.2 to $150 \mu \mathrm{g} / \mathrm{mL}$ ) of UCNPs, RB-UCNPs, Ce6-UCNPs, and RB/Ce6-UCNPs for $24 \mathrm{~h}$. The cytotoxicity of the samples was assessed by thiazolyl blue tetrazolium bromide (MTT, Sigma-Aldrich) cell proliferation assay. The MTT absorbance was measured using a spectrophotometer (Infinite 2000, Tecan) and $\mathrm{IC}_{50}$ was calculated using GraphPad Prism. To assess the cellular PDT effect, the cells with PS-UCNP uptake were irradiated for $10 \mathrm{~min}$ using the $808 \mathrm{~nm}$ CW laser $(\sim 600$ $\mathrm{mW}$ ) and incubated for $24 \mathrm{~h}$ (Xu et al., 2017a; Ding et al., 2018). The laser beam was focused at for different points in the same well for $10 \mathrm{~min}$ to illuminate the whole cells as much as possible (approximately $2.5 \mathrm{~min}$ of irradiation for each point). The cell survival was assessed using the MTT assay.

\section{Detection of ROS From PS-UCNPs in Live Cells}

The B16BL6 melanoma cells were seeded in a 96-well plate at a density of $5 \times 10^{3}$ cells per well. The UCNPs, RB-UCNPs, Ce6-UCNPs, and RB/Ce6-UCNPs were incubated with the cells for $24 \mathrm{~h}$, respectively. After irradiation by the $808 \mathrm{~nm}$ CW laser for $10 \mathrm{~min}$, the cells were incubated for $24 \mathrm{~h}$. The ROS in the live cells were measured using the DCFDA/H2DCFDA assay kit according to the manufacturer's instructions (Abcam). The one-way ANOVA test was used for the statistical analysis of significance, followed by a Tukey's $t$-test. $p$-values of $* * *<0.001$ were considered statistically significant.

\section{Fluorescence Imaging of ROS From PS-UCNPs in Live Cells}

To confirm the ROS generation, in vitro cellular studies were performed on the B16BL6 melanoma cells. The B16BL6 melanoma cells $\left(5 \times 10^{3}\right.$ cells per dish $)$ were seeded in a $35 \mathrm{~mm}^{2}$ glass-bottom dish and incubated for $48 \mathrm{~h}$. The cells were incubated with UCNPs, RB-UCNPs, Ce6-UCNPs, and RB/Ce6UCNPs for $24 \mathrm{~h}$, respectively, and irradiated using the $808 \mathrm{~nm}$ $\mathrm{CW}$ laser for $10 \mathrm{~min}$. After $24 \mathrm{~h}$, the cells were stained with CellROX deep red (5 $\mu \mathrm{M}$, Invitrogen), CellMask Green (1:1000, Invitrogen), and Hoechst 33352 (1:5000, Invitrogen). The nontreated cells were used as control. The fluorescence images were obtained using an LSM 780 confocal microscope (Carl Zeiss 780) with a water immersion $40 \times$ lens.

\section{Western Blot of Cellular Proteins}

The cellular protein expression was determined using western blot. The cellular protein was extracted using RIPA buffer (Abcam) and the concentrations were confirmed using the BCA assay kit (Thermo Fisher Scientific, Inc.). The electrophoresis was performed using $8 \%$ polyacrylamide gels (30 $\mu \mathrm{g} / \mathrm{lane})$. The gels were transferred to nitrocellulose membranes and incubated with a mouse monoclonal antibody against HSP70 (72 kDa, 1:1000, Invitrogen) and rabbit polyclonal antibody against HMGB1 (29 $\mathrm{kDa}, 1: 500$, Abcam), respectively. The mouse monoclonal antibody against GAPDH (36 kDa, 1:3000, Invitrogen) was used as the internal control. The signals were visualized using ECL and detected using the ImageQuant LAS 4000 system (GE Healthcare).

\section{Active Caspase-3 Colorimetric Assay}

The cell apoptosis was measured using the caspase-3 assay kit (Abcam). The B16BL6 melanoma cells were seeded in a 96-well plate at a density of $5 \times 10^{3}$ cells per well and the UCNPs, RBUCNPs, Ce6-UCNPs, and RB/Ce6-UCNPs were incubated for $24 \mathrm{~h}$, respectively. After irradiation with an $808 \mathrm{~nm}$ CW laser for $10 \mathrm{~min}$, the cells were incubated for $24 \mathrm{~h}$. The cells were lysed and centrifuged at $10,000 \times g$. The supernatant was collected and the assay was performed by following the manufacturer's instructions (Abcam). The one-way ANOVA test was used for the statistical analysis of significance, followed by a Tukey's $t$-test. $p$-values of $* * *<0.001$ were considered statistically significant.

\section{RESULTS AND DISCUSSION}

\section{Preparation and Characterization of PS-UCNPs}

The overall synthetic procedure for preparing PSUCNPs is illustrated in Figure 1. The highly crystalline $\mathrm{NaYF}_{4}: \mathrm{Yb}, \mathrm{Er}, \mathrm{Nd} @ \mathrm{NaYF}_{4}: \mathrm{Yb}, \mathrm{Nd}$ core@shell UCNPs were synthesized using a high-temperature thermal decomposition

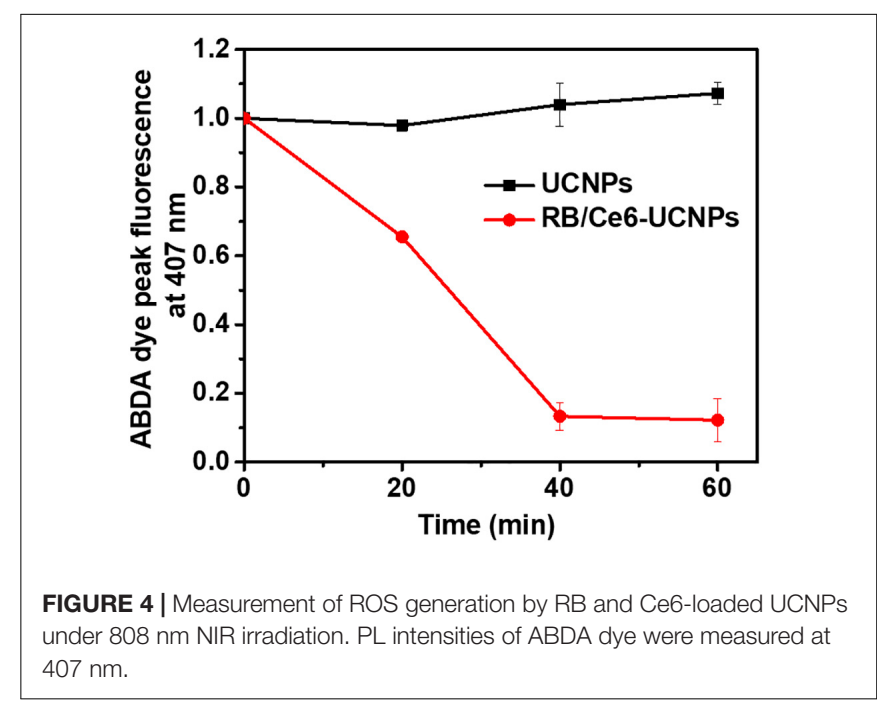




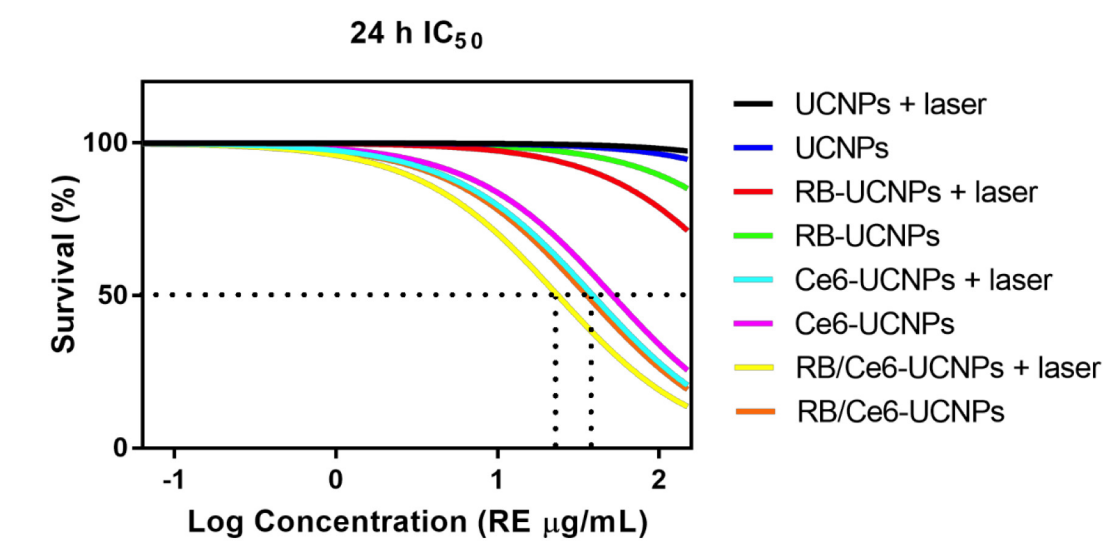

\begin{tabular}{|c|c|}
\hline & IC $_{50}(\mathrm{RE} \mu \mathrm{g} / \mathrm{mL})$ \\
\hline UCNPs + laser & N/A \\
\hline UCNPs & N/A \\
\hline RB-UCNPs + laser & 372.5 \\
\hline RB-UCNPs & 855.6 \\
\hline Ce6-UCNPs + laser & 38.89 \\
\hline Ce6-UCNPs & 51.52 \\
\hline RB/Ce6-UCNPs + laser & 23.64 \\
\hline RB/Ce6-UCNPs & 35.8 \\
\hline
\end{tabular}

FIGURE 5 | Cytotoxicity of UCNPs and PS-UCNPs in B16BL6 melanoma cells with or without 808 nm laser irradiation. The IC 50 values after 24 h of treatment were measured. The survivals were determined by the MTT assay using three replicated samples from three separate experiments. Results are presented as mean \pm SEM

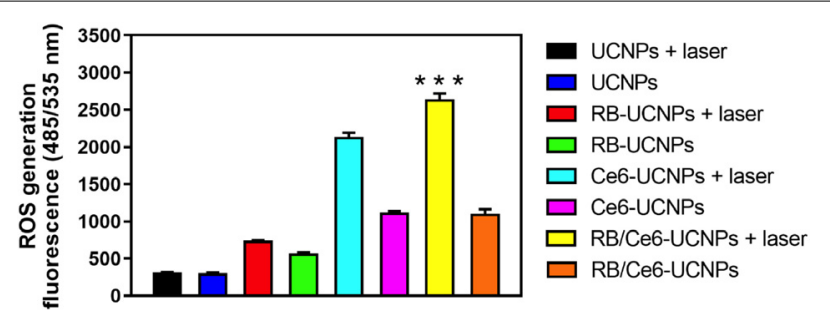

FIGURE 6 | Quantified ROS generation under laser irradiation in B16BL6 melanoma cells. The cellular ROS was measured using the DFCDA/H2DCFDA kit. The cells were incubated with each UCNP samples (100 RE $\mu \mathrm{g} / \mathrm{mL}$ ) for $3 \mathrm{~h}$ and then irradiated using an $808 \mathrm{~nm}$ NIR laser for $10 \mathrm{~min}$. All experiments were performed in triplication. The results are presented as mean \pm SEM. ${ }^{* * *} p<0.001$

method (Wang et al., 2010). The hydrophobic surface of the synthesized core@shell UCNPs was converted to hydrophilic by DSPE-PEG encapsulation (Park et al., 2012). DSPE-PEG is an amphiphilic consisting of a hydrophilic PEG chain and hydrophobic hydrocarbon chain. The hydrophobic UCNPs were encapsulated by DSPE-PEG through a hydrophobic interaction between the surface oleic acid and DSPE. The hydrophobic photosensitizers, Ce6 and RB, were also loaded in the hydrophobic layer (He et al., 2018).

To reduce the overheating effect of the $980 \mathrm{~nm}$ NIR light source, $\mathrm{Nd}$ ions were added to the core UCNPs with $\mathrm{Yb}$ ions as a sensitizer (Wang et al., 2013). The $808 \mathrm{~nm}$ NIR photons were absorbed by the $\mathrm{Nd}$ ions and transferred to the $\mathrm{Yb}$ ions for upconversion luminescence (Supplementary Figure S1). Continuous laser irradiation resulted in almost no increase in the temperature of the UCNP sample (Supplementary Figure S2). To improve the upconversion emission efficiency, the shell was grown on the core UCNPs to minimize non-radiative decay on the surface. Unlike the conventional inert shell without any sensitizers (e.g., $\mathrm{NaYF}_{4}$ or $\mathrm{NaGdF}_{4}$ ), the active shell including $\mathrm{Yb}$ and $\mathrm{Nd}$ ions was selected for more efficient $808 \mathrm{~nm}$-triggered upconversion luminescence (Huang and Lin, 2015).
The synthesized $\mathrm{NaYF}_{4}: \mathrm{Yb}, \mathrm{Er}, \mathrm{Nd}$ core UCNPs were $18 \mathrm{~nm}$ in diameter with a uniform spherical shape (Figure 2a). After the epitaxial growth on the $\mathrm{NaYF}_{4}: \mathrm{Yb}, \mathrm{Nd}$ shell of the core UCNPs, the core@shell UCNPs had a uniform size of $22 \mathrm{~nm}$ (Figure 2b). The uniform core@shell UCNPs with approximately $4 \mathrm{~nm}$ size increment demonstrated the successful synthesis of core@shell UCNPs. The XRD patterns of the UCNPs were well-matched with the hexagonal phase of the $\beta-\mathrm{NaYF}_{4}$ crystals (JCPDS No. 016-0334) (Figure 2c). The composition of the UCNPs was also measured using ICP-AES (Supplementary Table S1). The core and core@shell UCNPs had a similar molar ratio of the lanthanide elements to the theoretical values, indicating successful synthesis of core@shell UCNPs.

The upconversion emission spectra of UCNPs were obtained with an $808 \mathrm{~nm}$ excitation $\left(500 \mathrm{~mW} / \mathrm{cm}^{2}\right)$ (Figure 3A). The active shell growth enhanced the overall emission intensity by approximately 60 times. As shown in the elemental analysis (Supplementary Table S1), more Nd ions were doped into the shell to collect more $808 \mathrm{~nm}$ NIR photons, which may affect the significant increase in luminescence efficiency. The green emission at $520 \mathrm{~nm}\left({ }^{2} \mathrm{H}_{11 / 2}{ }^{4} \mathrm{I}_{15 / 2}\right)$ and $539 \mathrm{~nm}\left({ }^{4} \mathrm{~S}_{3 / 2}{ }^{4} \mathrm{I}_{15 / 2}\right)$, the red emission at $653 \mathrm{~nm}\left({ }^{4} \mathrm{~F}_{9 / 2}{ }^{4} \mathrm{I}_{15 / 2}\right)$, and the blue emission at $407 \mathrm{~nm}\left({ }^{2} \mathrm{H}_{9 / 2}{ }^{4} \mathrm{I}_{15 / 2}\right)$ were observed, respectively (Figure 3A and Supplementary Figure S1).

Chlorin e6 and RB were selected as the photosensitizers as their absorption wavelengths were well-matched with the emission wavelengths of UCNPs. Figure $\mathbf{3 B}$ shows that the absorption of Ce6 overlaps with the blue and red emission of UCNPs, and that of RB overlaps with the green emission of UCNPs. Regardless of the type of photosensitizers, the upconversion emission of PS-UCNPs was significantly reduced after photosensitizer loading (Figure 3C and Supplementary Figure S3). The red and blue emission shows an obvious decrease for Ce6-UCNPs, while the green emission significantly decreased for RB-UCNPs. This indicates that the emissions of the UCNPs have been absorbed by the photosensitizers, and the energy transfer between the photosensitizers and UCNPs was successful. 


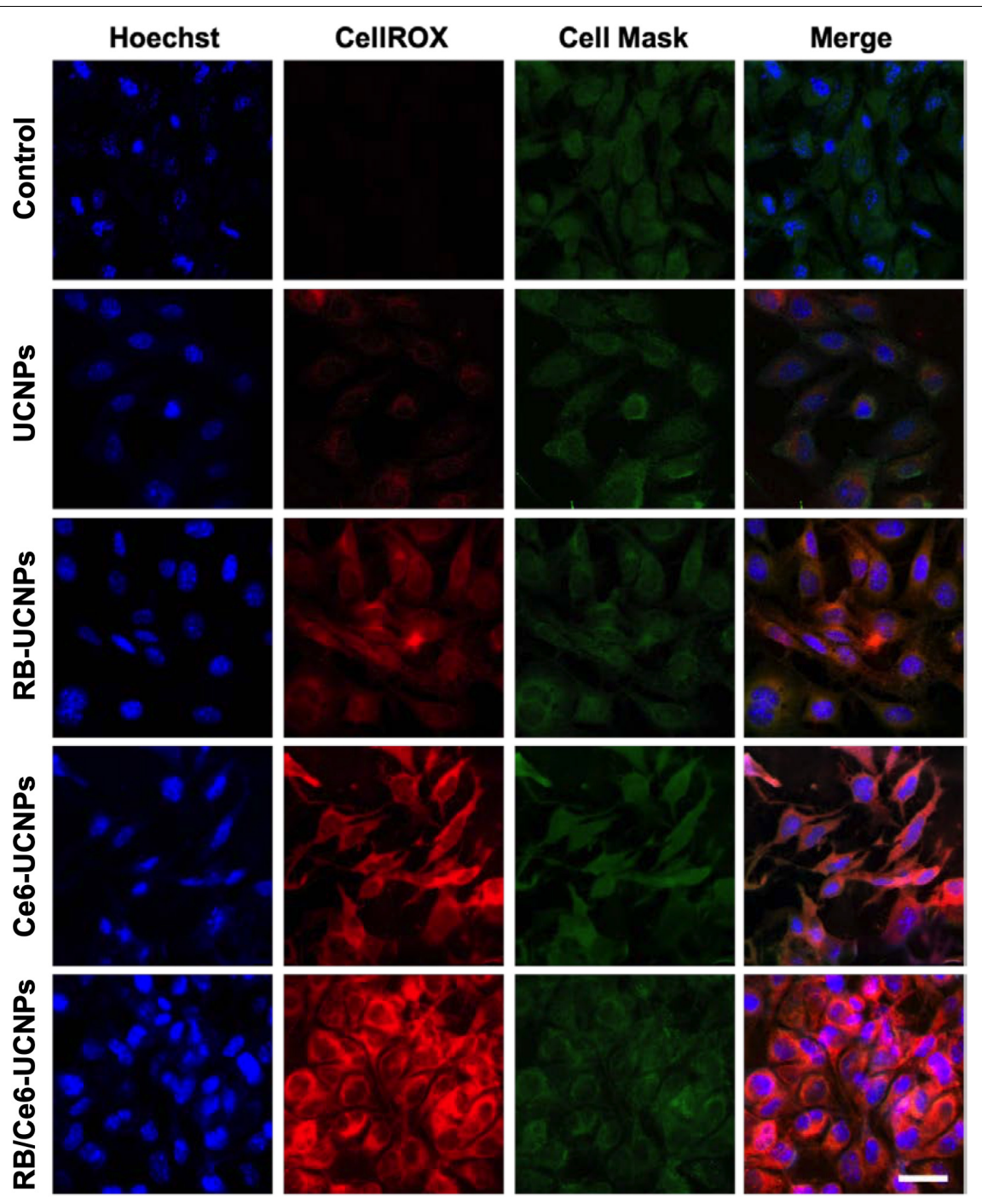

FIGURE 7 | Representative confocal images of laser mediated ROS generation in B16BL6 melanoma cells. The cells were incubated with each UCNP samples (100 $\mathrm{RE} \mu \mathrm{g} / \mathrm{mL}$ ) for $3 \mathrm{~h}$ and then irradiated using an $808 \mathrm{~nm}$ NIR CW laser for $10 \mathrm{~min}$. All experiments were performed in triplication. The scale bar is $25 \mu \mathrm{m}$.

The production of ROS by PS-UCNPs was evaluated using ABDA (Lindig et al., 1980; Shen et al., 2011). ABDA selectively reacts with singlet oxygen and decomposes to decrease the fluorescence. Compared to the control sample without a photosensitizer, the fluorescence intensity in each sample decreases (Supplementary Figure S3). This means that ROS were generated by the $808 \mathrm{~nm}$ NIR excitation. Among them, the fluorescence intensity in RB/Ce6-UCNPs significantly decreases, indicate that UCNPs with dual photosensitizers were more efficient in ROS production and have improved PDT efficiency (Figure 4).

\section{Cytotoxicity and Cellular PDT Effect of PS-UCNPs}

The cytotoxic effects of various treatments were evaluated in B16BL6 mouse melanoma cells using the MTT assay.
A dosage of $5.6 \mu \mathrm{g} / \mathrm{mL}$ of RB/Ce6-UCNPs showed a $92 \%$ survival rate for $24 \mathrm{~h}$ incubation. However, the survival rate significantly decreased to $30 \%$ at $50 \mu \mathrm{g} / \mathrm{mL}$ of RB/Ce6-UCNPs (Supplementary Figure S4a). Compared to RB/Ce6-UCNPs, the UCNP and RB-UCNP treatments had no significant effect on the cell survival rate up to $100 \mu \mathrm{g} / \mathrm{mL}$ (Figure 5 and Supplementary Figure S5). The cytotoxicity of RB/Ce6UCNPs without laser irradiation seems to be due to Ce6 released from the UCNPs. Ce6-UCNPs also showed a similar cytotoxic effect as RB/Ce6-UCNPs. When the B16BL6 melanoma cells were treated with RB/Ce6-UCNPs and $808 \mathrm{~nm} \mathrm{CW}$ laser irradiation for $10 \mathrm{~min}$, the cell viability was further decreased to $84 \%$ at $5.6 \mu \mathrm{g} / \mathrm{mL}$ and $24 \%$ at $50 \mu \mathrm{g} / \mathrm{mL}$ (Supplementary Figure S4b). Figure 5 shows that the laser irradiation resulted in lower $\mathrm{IC}_{50}$ values than non-laser irradiated treatment regardless of the combination of the photosensitizers. Among them, the 


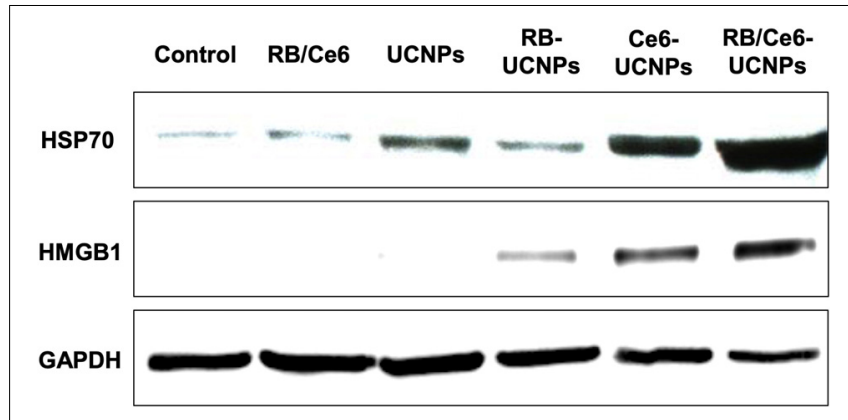

FIGURE 8 | Cellular protein expression of HSP70 $(70 \mathrm{kDa})$ and HMGB1 (29 kDa). The B16BL6 melanoma cells were incubated with each UCNP samples (100 RE $\mu \mathrm{g} / \mathrm{mL}$ ) for $3 \mathrm{~h}$ and then irradiated using an $808 \mathrm{~nm}$ NIR CW laser for $10 \mathrm{~min}$. Ten spots were selected and irradiated in each well. GAPDH $(38 \mathrm{kDa})$ was used as an internal control. All experiments were performed in triplication.

RB/Ce6-UCNPs with laser irradiation killed the B16BL6 cells more effectively than single photosensitizer-loaded UCNPs. Accordingly, $16.7 \mu \mathrm{g} / \mathrm{mL}$ of RB/Ce6-UCNPs and $10 \mathrm{~min}$ irradiation were selected as the conditions for the following PDT experiments without significant cytotoxicity of the photosensitizer itself.

\section{PDT-Induced Intracellular ROS Generation and Apoptosis}

The activated photosensitizer under laser irradiation can generate ROS, which can destroy the tumor cells through the apoptosis or necrosis process (Yang and Wang, 2013). The ability of UCNPs to generate ROS was evaluated using the DCFDA/H2DCFDA assay kit in B16BL6 melanoma cells. As shown in Figure 6, the $808 \mathrm{~nm}$ laser irradiation allows PS-UCNPs to increase ROS generation than that without laser irradiation. In particular, the laser-irradiated $\mathrm{RB} / \mathrm{Ce} 6$ UCNPs showed significantly higher ROS generation in the experimental group. Similar to the DCFDA/H2DCFDA assay, the intracellular ROS generation by PS-UCNPs was confirmed using a fluorogenic probe, CellRox deep red, in live B16BL6 melanoma cell imaging (Figure 7 ). The deep red signal by $\mathrm{RB} / \mathrm{Ce} 6-\mathrm{UCNP}$ was much stronger than that treated with RB-UCNPs or Ce6-UCNPs, indicating a very efficient ROS generation in cells.

The PDT-induced oxidative stress resulted in extended tumor apoptosis (Yang et al., 2016). The damaged or dying tumor cells can be characterized by detecting damage-associated molecular patterns (DAMPs), such as heat shock protein 70 (HSP70) (Korbelik et al., 2005) and high mobility group box 1 (HMGB1) (Panzarini et al., 2013). In particular, HSPs have been known to inhabit in all blood cells and migrate to the cell surface membrane during apoptosis induced by PDT (Zhou et al., 2008; Yang et al., 2016). The expression of HSP70 and HMGB1 protein by PDT was confirmed by western blot. The laserirradiated RB/Ce6-UCNPs significantly increased the expression levels of HSP70 and HMGB1 protein in B16BL6 melanoma cells (Figure 8).

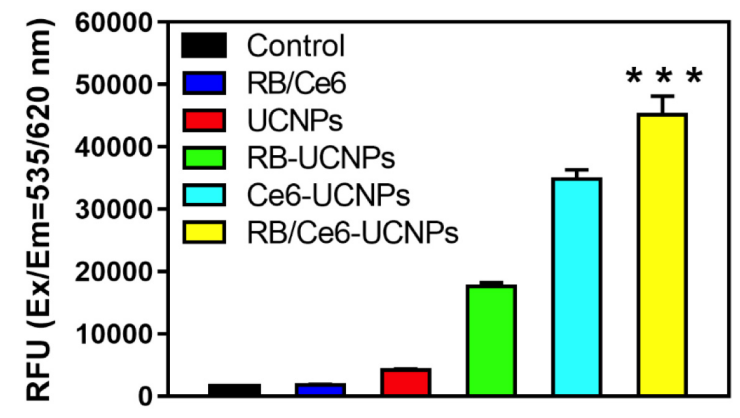

FIGURE 9 | Cellular caspase-3 activity was measured using the fluorometric kit. The B16BL6 melanoma cells were cultured with each UCNP samples (100 $\mathrm{RE} \mu \mathrm{g} / \mathrm{mL}$ ) for $3 \mathrm{~h}$ and irradiated using an $808 \mathrm{~nm}$ NIR CW laser for $10 \mathrm{~min}$. Ten spots were selected and irradiated in each well. The extracted cell proteins were confirmed according to the manufacturer's instruction. All experiments were performed in triplication. The results are presented as mean \pm SEM. ${ }^{\star \star \star} p<0.001$.

The PDT can induce apoptosis or necrosis, or a combination thereof, although PDT is highly efficient at inducing apoptosis mainly (Oleinick and Evans, 1998). This study investigated the active caspase-3 downstream apoptotic marker using a colorimetric assay kit. Figure 9 shows a significant increase in caspase-3 activity when a laser is irradiated to RB/Ce6-UCNPs treated cells. The RB/Ce6-UCNPs showed activated caspase-3 levels of 1.3 and 2.7 times higher than Ce6-UCNPs and RBUCNPs, respectively. These results demonstrate that dual PS-UCNPs show high efficacy and a synergistic effect in PDT therapy.

\section{CONCLUSION}

In this study, UCNPs with dual photosensitizers were developed as a highly efficient NIR-triggered PDT agent. An $808 \mathrm{~nm}$ excitation wavelength was used to minimize overheating by the NIR light source. As the addition of the energy transfer step of UCNPs by Nd ions reduces the upconversion efficiency, a core@shell structure with an active shell was prepared. The use of dual photosensitizers for the red and green emission of UCNPs improved the ROS generation efficiency. A synergistic ROS generation of PS-UCNPs was studied in cellular studies and the PDT-induced apoptosis was confirmed. However, the leakage of PS from the UCNPs, in particular Ce6, seems to induce cytotoxicity. Therefore, it is necessary to improve the PS loading method to minimize PS leakage and ensure long term stability.

\section{DATA AVAILABILITY STATEMENT}

All datasets generated for this study are included in the article/Supplementary Material. 


\section{AUTHOR CONTRIBUTIONS}

SYL, RL, SL, and YP designed the research and wrote the manuscript with inputs from EK. SYL synthesized and characterized the PS-UCNPs under the guidance of YP. RL, EK, and SL performed the cell experiments.

\section{FUNDING}

This work was supported by the National Research Foundation of Korea (NRF) grant funded by the Korea Government (Ministry of Science, ICT \& Future Planning) (Nos. 2017R1C1B1010703 and 2019R1A4A2001527), Korea Institute of Science and Technology (KIST) Institutional Program (2E30180), and International

\section{REFERENCES}

Chen, G. Y., Qju, H. L., Prasad, P. N., and Chen, X. Y. (2014). Upconversion nanoparticles: design, nanochemistry, and applications in theranostics. Chem. Rev. 114, 5161-5214. doi: 10.1021/cr400425h

Ding, B. B., Shao, S., Yu, C., Teng, B., Wang, M. F., Cheng, Z. Y., et al. (2018). Largepore mesoporous-silica-coated upconversion nanoparticles as multifunctional immunoadjuvants with ultrahigh photosensitizer and antigen loading efficiency for improved cancer photodynamic immunotherapy. Adv. Mater. 30:10. doi: 10.1002/adma.201802479

Dolmans, D., Fukumura, D., and Jain, R. K. (2003). Photodynamic therapy for cancer. Nat. Rev. Cancer 3, 380-387.

Dong, H., Du, S. R., Zheng, X. Y., Lyu, G. M., Sun, L. D., Li, L. D., et al. (2015). Lanthanide nanoparticles: from design toward bioimaging and therapy. Chem. Rev. 115, 10725-10815. doi: 10.1021/acs.chemrev.5b00091

Feng, Y. S., Wu, Y. N., Zuo, J., Tu, L. P., Que, I., Chang, Y. L., et al. (2019). Assembly of upconversion nanophotosensitizer in vivo to achieve scatheless real-time imaging and selective photodynamic therapy. Biomaterials 201, 33-41. doi: 10.1016/j.biomaterials.2019.02.015

Guo, H. C., Qian, H. S., Idris, N. M., and Zhang, Y. (2010). Singlet oxygeninduced apoptosis of cancer cells using upconversion fluorescent nanoparticles as a carrier of photosensitizer. Nanomed. Nanotechnol. Biol. Med. 6, 486-495. doi: 10.1016/j.nano.2009.11.004

He, S., Johnson, N. J. J., Huu, V. A. N., Huang, Y. R., and Almutairi, A. (2018). Leveraging spectral matching between photosensitizers and upconversion nanoparticles for $808 \mathrm{~nm}$-activated photodynamic therapy. Chem. Mater. 30, 3991-4000. doi: 10.1021/acs.chemmater.7b04700

Huang, W. T., Chan, M. H., Chen, X. Y., Hsiao, M., and Liu, R. S. (2020). Theranostic nanobubble encapsulating a plasmon-enhanced upconversion hybrid nanosystem for cancer therapy. Theranostics 10, 782-796. doi: 10.7150/ thno.38684

Huang, X. Y., and Lin, J. (2015). Active-core/active-shell nanostructured design: an effective strategy to enhance $\mathrm{Nd} 3+/ \mathrm{Yb} 3+$ cascade sensitized upconversion luminescence in lanthanide-doped nanoparticles. J. Mater. Chem. C 3, $7652-$ 7657. doi: 10.1039/c5tc01438g

Hwang, H. S., Shin, H., Han, J., and Na, K. (2018). Combination of photodynamic therapy (PDT) and anti-tumor immunity in cancer therapy. J. Pharm. Investig. 48, 143-151. doi: 10.1007/s40005-017-0377-x

Idris, N. M., Gnanasammandhan, M. K., Zhang, J., Ho, P. C., Mahendran, R., and Zhang, Y. (2012). In vivo photodynamic therapy using upconversion nanoparticles as remote-controlled nanotransducers. Nat. Med. 18, 1580U1190. doi: 10.1038/nm.2933

Kobayashi, H., and Choyke, P. L. (2019). Near-infrared photoimmunotherapy of cancer. Acc. Chem. Res. 52, 2332-2339. doi: 10.1021/acs.accounts.9b00273

Korbelik, M., Sun, J. H., and Cecic, I. (2005). Photodynamic therapy-induced cell surface expression and release of heat shock proteins: relevance for tumor response. Cancer Res. 65, 1018-1026.
Research Organization for Advanced Science and Technology (IROAST) (Grant Number 005-5800100106).

\section{ACKNOWLEDGMENTS}

We would like to thank the Editage (www.editage.co.kr) for English language editing.

\section{SUPPLEMENTARY MATERIAL}

The Supplementary Material for this article can be found online at: https://www.frontiersin.org/articles/10.3389/fbioe. 2020.00275/full\#supplementary-material

Lindig, B. A., Rodgers, M. A. J., and Schaap, A. P. (1980). Determination of the lifetime of singlet oxygen in water-d2 using 9,10-anthracenedipropionic acid, a water-soluble probe. J. Am. Chem. Soc. 102, 5590-5593. doi: 10.1021/ ja00537a030

Maeding, N., Verwanger, T., and Krammer, B. (2016). Boosting tumor-specific immunity using PDT. Cancers 8:14.

Oleinick, N. L., and Evans, H. H. (1998). The photobiology of photodynamic therapy: cellular targets and mechanisms. Radiat. Res. 150, S146-S156.

Panzarini, E., Inguscio, V., and Dini, L. (2013). Immunogenic cell death: can it be exploited in photodynamic therapy for cancer?. Biomed. Res. Int. 2013:482160.

Park, Y., Kim, H. M., Kim, J. H., Moon, K. C., Yoo, B., Lee, K. T., et al. (2012). Theranostic probe based on lanthanide-doped nanoparticles for simultaneous in vivo dual-modal imaging and photodynamic therapy. Adv. Mater. 24, 57555761. doi: 10.1002/adma.201202433

Park, Y. I., Kim, J. H., Lee, K. T., Jeon, K. S., Bin, N. H., Yu, J. H., et al. (2009). Nonblinking and nonbleaching upconverting nanoparticles as an optical imaging nanoprobe and T1 magnetic resonance imaging contrast agent. Adv. Mater. 21, 4467-4480.

Shen, X. Q., Li, L., Wu, H., Yao, S. Q., and Xu, Q. H. (2011). Photosensitizerdoped conjugated polymer nanoparticles for simultaneous two-photon imaging and two-photon photodynamic therapy in living cells. Nanoscale 3, 5140-5146. doi: $10.1039 / \mathrm{cln} r 11104 \mathrm{c}$

Tsai, Y. C., Vijayaraghavan, P., Chiang, W. H., Chen, H. H., Liu, T. I., Shen, M. Y., et al. (2018). Targeted delivery of functionalized upconversion nanoparticles for externally triggered photothermal/photodynamic therapies of brain glioblastoma. Theranostics 8, 1435-1448. doi: 10.7150/thno. 22482

Wang, F., Wang, J. A., and Liu, X. G. (2010). Direct evidence of a surface quenching effect on size-dependent luminescence of upconversion nanoparticles. Angew. Chem. Int. Ed. 49, 7456-7460. doi: 10.1002/anie.201003959

Wang, Y. F., Liu, G. Y., Sun, L. D., Xiao, J. W., Zhou, J. C., and Yan, C. H. (2013). $\mathrm{Nd} 3+$-sensitized upconversion nanophosphors: efficient in vivo bioimaging probes with minimized heating effect. Acs Nano 7, 7200-7206. doi: 10.1021/ nn402601d

Wu, S. W., Han, G., Milliron, D. J., Aloni, S., Altoe, V., Talapin, D. V., et al. (2009). Non-blinking and photostable upconverted luminescence from single lanthanide-doped nanocrystals. Proc. Natl. Acad. Sci. U.S.A. 106, 10917-10921. doi: 10.1073/pnas.0904792106

Xu, J. T., Xu, L. G., Wang, C. Y., Yang, R., Zhuang, Q., Han, X., et al. (2017a). Nearinfrared-triggered photodynamic therapy with multitasking upconversion nanoparticles in combination with checkpoint blockade for immunotherapy of colorectal cancer. ACS Nano 11, 4463-4474. doi: 10.1021/acsnano.7b 00715

Xu, J. T., Yang, P. P., Sun, M. D., Bi, H. T., Liu, B., Yang, D., et al. (2017b). Highly emissive dye-sensitized upconversion nanostructure for dual-photosensitizer photodynamic therapy and bioimaging. ACS Nano 11, 4133-4144. doi: 10.1021/ acsnano.7b00944 
Yan, S. Q., Zeng, X. M., Tang, Y. A., Liu, B. F., Wang, Y., and Liu, X. G. (2019). Activating antitumor immunity and antimetastatic effect through polydopamine-encapsulated core-shell upconversion nanoparticles. Adv. Mater. 31:8. doi: 10.1002/adma.201905825

Yang, M., Wang, H., Wang, Z. H., Han, Z. H., and Gu, Y. Q. (2019). A Nd3+ sensitized upconversion nanosystem with dual photosensitizers for improving photodynamic therapy efficacy. Biomater. Sci. 7, 1686-1695. doi: 10.1039/ c8bm01570h

Yang, Y. M., Hu, Y., and Wang, H. J. (2016). Targeting antitumor immune response for enhancing the efficacy of photodynamic therapy of cancer: recent advances and future perspectives. Oxidat. Med. Cell. Longev. 2016:5274084.

Yang, Y. M., and Wang, H. J. (2013). Perspectives of nanotechnology in minimally invasive therapy of breast cancer. J. Healthc. Eng. 4, 67-86. doi: 10.1260/20402295.4.1.67

Zheng, Y. H., Yin, G. F., Le, V., Zhang, A. L., Chen, S. Y., Liang, X., et al. (2016). Photodynamic-therapy activates immune response by disrupting immunity homeostasis of tumor cells, which generates vaccine for cancer therapy. Int. J. Biol. Sci. 12, 120-132. doi: 10.7150/ijbs.12852

Zhou, F., Xing, D., and Chen, W. R. (2008). Dynamics and mechanism of HSP70 translocation induced by photodynamic therapy treatment. Cancer Lett. 264, 135-144. doi: 10.1016/j.canlet.2008.01.040

Conflict of Interest: The authors declare that the research was conducted in the absence of any commercial or financial relationships that could be construed as a potential conflict of interest.

Copyright (c) 2020 Lee, Lee, Kim, Lee and Park. This is an open-access article distributed under the terms of the Creative Commons Attribution License (CC BY). The use, distribution or reproduction in other forums is permitted, provided the original author(s) and the copyright owner(s) are credited and that the original publication in this journal is cited, in accordance with accepted academic practice. No use, distribution or reproduction is permitted which does not comply with these terms. 